\title{
QUALITY ANALYSIS AND IMPROVEMENT OF FUNDAMENTAL GEOGRAPHIC NATIONAL CONDITIONS MONITORING RESULTS
}

\author{
Miao LI ${ }^{1, *}$, HaiPeng CHEN ${ }^{1}$, ZongBiao TIAN ${ }^{1}$, Bo QIU ${ }^{1}$, WenJun XIE ${ }^{1}$, YuHeng CHEN $^{1}$ \\ ${ }^{1}$ National Quality Inspection and Testing Center for Surveying and Mapping Products, Beijing, China - $9105513 @ q q . c o m$
}

\author{
Commission III, ICWG III/IVb
}

KEY WORDS: Fundamental Geographical National Monitoring, Quality Requirements, Problem-oriented, Quality Control, Big Data

\begin{abstract}
:
With the integration and unification of natural resources, the application fields and application levels of geographic national condition monitoring results have also expanded and improved, and the data quality requirements for national condition monitoring have become higher and higher, especially the results of fundamental geographic national conditions monitoring, the quality of the results is the basis of monitoring and the life of monitoring. Based on the recent practice in fundamental geographic national conditions monitoring, this paper summarizes the quality requirements of monitoring results, analyzes typical quality problems and quality influencing factors, and three measures for improving the quality of the results are proposed: one is a problem-oriented quality inspection method; the other is the key points of quality control in the fundamental national condition monitoring process; the third is the preliminary exploration of the application of big data in monitoring.
\end{abstract}

\section{INTRODUCTION}

Geography national conditions are important basic national conditions. Doing a good job of dynamic monitoring of major national conditions and national strength is the basic work to comprehensively understand national conditions, grasp national conditions, and formulate national policies (Li, D.R., 2018). In particular, with the integration and unification of natural resource supervision, the application field of geographic national conditions monitoring results has expanded and the application level has also increased(Chen, H.Q., 2018). The geographic national conditions monitoring results will continue to play a fundamental role in supporting various types of natural resource surveys Function, and the quality level of its results will directly affect the accuracy and reliability of natural resources survey and monitoring of various statistics and analysis results(Yang, Y., 2019). The natural resources survey and monitoring business puts forward higher requirements on the results of geographic national conditions monitoring. Therefore, the research on the method of improving the quality of basic geographic national conditions monitoring results is its important guarantee. This paper analyzes and summarizes the quality requirements of the basic geographical and national conditions monitoring results under the unified pattern of natural resource supervision and integration, and analyzes the quality influencing factors in combination with the frequent and critical typical problems in actual production, and proposes methods and measures for quality improvement .

\section{QUALITY REQUIREMENTS}

The results of geographic national condition monitoring mainly use the rich spectrum and texture and time phase features contained in multi-source aerospace remote sensing images(Shi, W.Z., 2013), combined with various reference materials and knowledge, using interactive interpretation, field survey and other methods to extract the surface coverage and change information, using data sets, metadata, and statistics for data expression, with quality characteristics such as mathematical foundation, data accuracy and up-to-date. Due to the vast territory of our country, the natural and human environments vary greatly from place to place, and satellite remote sensing image sources often have large differences in sensor multisources, which has an adverse effect on the collection of surface change information. And software factors, it is inevitable that there are various quality problems in the results of geographic national conditions monitoring data. Among them, time accuracy, position accuracy, classification accuracy and attribute accuracy seriously affect the data quality of the monitoring results. In the process of integration and fusion of natural resources-oriented big data, there are risks that affect the application of statistical analysis by government departments and the accuracy of decision-making.

\subsection{Time Accuracy}

The results of geographic national conditions monitoring data require that the original data sources, thematic materials, and results data meet the requirements of the time(Li, Y.L., 2017) . Generally use data verification and analysis methods for inspection, the method of data verification and analysis is used for the content of verification includes the use of temporal information of image data sources, the current status of thematic data in various industries, the timeliness of field surveys and the time point of final results.

\subsection{Position Accuracy}

The mathematical accuracy of the monitoring results is mainly reflected in the correspondence between the vector position and

\footnotetext{
* Corresponding author
} 
the features on the image. The contents of the inspection include geometric displacement and edge connection. In general, it is required that on the basis of qualified images, the position accuracy of the surface coverage classification boundaries with obvious boundaries on the image, the boundaries of element and the position accuracy of location point points should be controlled within 5 pixels. Under special circumstances, such as shielding and shading of high-rise buildings, the position accuracy should be controlled within 10 pixels in principle. After the edge is connected, the graphics data should be smooth and continuous, avoid hard folds and sharp corners, and ensure that the edges are within 0.01 meters of the boundary. In addition, the topological consistency between the layers of the monitoring results is required.

\subsection{Classification Accuracy}

The contents of the classification accuracy check of the monitoring results of geographical conditions mainly include the correctness of classification and the completeness of coverage. First of all, it is required that the data range should completely cover the scope of the monitoring task area. Secondly, in the transition zone without obvious boundaries, the surface coverage classification data should at least ensure that they meet the classification requirements of the previous level. Objects with obvious boundaries on the image should be correctly classified in strict accordance with the classification requirements.

\subsection{Attribute Accuracy}

The attribute accuracy check of the geographical national conditions monitoring results mainly includes the completeness and correctness of the general attribute items and the proprietary attribute items. The range of the value is required to meet the requirement, the attributes of the change type are objective and correct, and the attributes of the update object are consistent with the attributes of the DOM, field survey results, thematic data and other reference materials.

\section{TYPICAL PROBLEM ANALYSIS}

\subsection{Data Source Usage Problems}

Incorrect use of the data source will cause quality problems in terms of time accuracy, position accuracy, classification accuracy, and attribute accuracy. The collection of data sources mainly includes image data and thematic data of professional departments. The state distributes the images several times in batches, while allowing local governments to cooperate in image collection. In the collection of thematic data, in addition to the national basic data, there are also thematic data collected by the relevant departments of water conservancy, forestry, land transportation, civil affairs, environment and so on by the provinces themselves. In the process of using the data source, it is necessary to comprehensively consider the requirements of image resolution and shooting time, and it is necessary to make full use of the current and well-regulated data of authoritative professional departments. The following problems are common in the use of data sources: (1) Multi-source images are covered in the same important area, and high-resolution images are not used for data production, resulting in excessive collection accuracy. (2) The same area has different time-phase images of the same sensor, The April-June image or the latest image is not used for production, and the production uses the earlier image, resulting in missing updates. (3) Other kinds of basic surveying and mapping results and the results of thematic data of water conservancy, forestry, land, transportation, civil affairs, environment and other related departments are not used correctly or uniformly, resulting in the inconsistency between the attributes of factors and entities.

\subsection{Position Update Problems}

3.2.1 Position Accuracy Exceeds the Limit: The problem of excessive accuracy of position is mainly reflected in the use of images. For example, in urban areas with supplementary highresolution commercial satellite images as the main image source for production, low-resolution images were used for range acquisition in the early stage, during the monitoring period, boundary adjustment was required based on high-resolution image, but the operators did not follow the requirements, it causes the position reference monitoring image to exceed the error limit. There is also a case where there is a significant change compared with the two phase images, the object changes with expansion, and the boundary range is not updated according to the images of the new period, resulting in the inconsistency between the boundary range of the object and the image, the category and scope of ground objects have been changed, only the object code is modified according to the images of the new period, but the scope is not modified, resulting in the position accuracy does not meet the requirements. Figure 1 reflects the problem that the acquisition accuracy of the new construction site exceeds the limit, only the category code is modified without modifying boundary according to the image, which leads to the boundary exceeds the limit and is not inconsistent with the image.

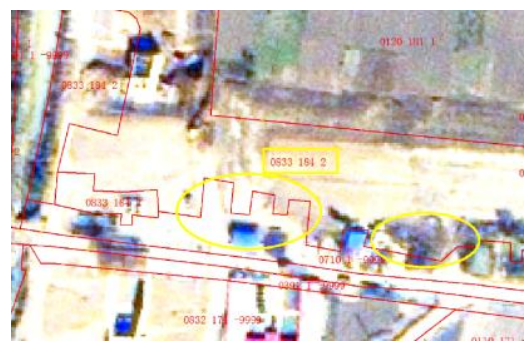

Figure 1. Position accuracy exceeded

3.2.2 Object Missing Update: In the production process, due to insufficient verification of the technical route and inconsistent understanding of the updated standards, the objects are missing to update. Non-standard production only use the background vector data and the monitoring period image to collect the change information in the identified change area, the risk of this operation mode is that the operator thinks that the two periods image does not change, and ignore errors that miss updates in earlier version data resulting in missed updates to this changed area. The ground features have the same spectral characteristics and texture characteristics, and meet the updated area requirements, due to different operators' inability to understand the definition, classification and collection norm of certain features, the update scale is inconsistent, resulting in objects are not updated. In the process of checking the results of the national conditions monitoring data, the investigation of the large area of misclassification of ground objects is the key step. Due to the omission and update error of ground objects, the statistical analysis and use of data volume on monitoring data change will be directly affected. Figure 2 reflects that the image contrast of the two phases has changed significantly, and 
the ground object has changed from the farmland to the building area, and the data has not been updated.

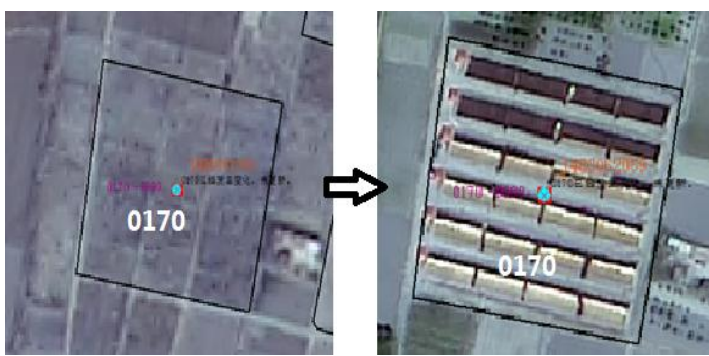

Figure 2. Object not updated

3.2.3 Constraint Relation Problems: The completion of a unit of results may have more than one operator to complete, which is not to do the change information linkage update hidden trouble. The location of the national conditions elements was not modified when modifying the surface coverage boundary, or the feature objects of other layers were not simultaneously modified when modifying the national condition feature objects, resulting in an incorrect constraint relationship between the objects. Figure 3 reflects the problem that the pavement range changes in the surface coverage, but the roads in the data set of national conditions are not updated. Figure 4 reflects that the water surface range in the surface coverage has been modified, but the high water boundary of the data set of national conditions has not been modified synchronously.
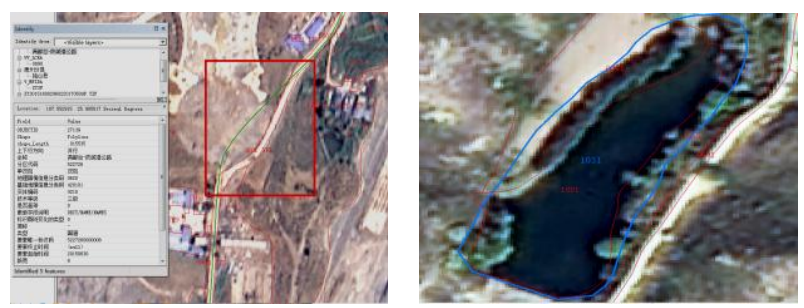

Figure 3. Road constraint error Figure 4. River constraint error

\subsection{Attribute Value Problems}

3.3.1 Enumeration Value Error: There are some enumerated fields in the monitoring data, such as change type, $\mathrm{GB}$, featID, etc., in order to improve production efficiency, some attribute items will be automatically assigned by software in order to improve production efficiency. However, production software has insufficient testing and verification, and it is inevitable that there are some attribute assignment errors caused by human and software defects. The field of change type reflects the change in the results of geographical national conditions monitoring data, the value should be assigned as $1,2,4,-2,-3$ or 9 according to the specific situation, it is required that the element unique identification code of the new element should be empty or the default value, only objects whose scope has changed require that the element unique identifier be used before. In the production process, there are attribute errors caused by personal incomprehension, and there are also value errors caused by the software being put into use without testing and verification. Figure 5 shows that the problem is the change type attribute value error, the water area has changed, and the change type field is assigned a value of 2 .

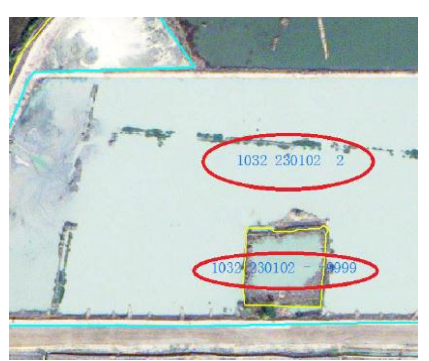

Figure 5. Change type error

3.3.2 Proprietary attribute Value Error: There are three problems in production: The first, it does not make full use of the information of thematic data, and the attribute of the thematic data is not filled in. The second, it fully relies on the thematic data, does not analyze, sort out and check the thematic data, and does not update the obvious errors, resulting in the inconsistency between the attributes of the object and the thematic data. The third, no proprietary attribute values were filled in according to field survey results. Figure 6 reflects that according to the interpretation of sample photo, the number of newly added road lanes and road width attributes are inconsistent with the photo. Figure 7 shows that the new forest farm did not assign an area field value based on the thematic data.

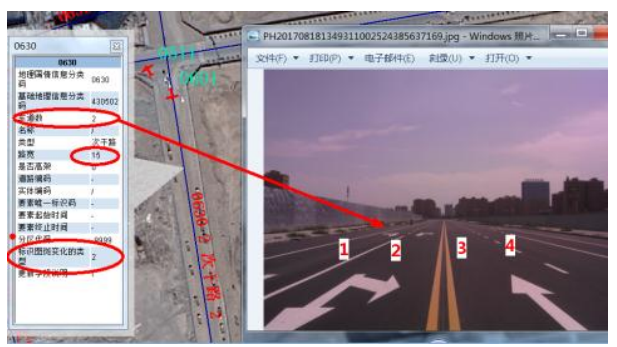

Figure 6. Lane error

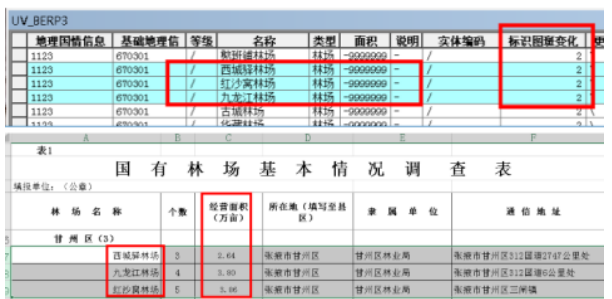

Figure 7. Area attribute error

3.3.3 Classification Error: Large areas update errors will directly lead to unqualified data results, reduce data credibility, and seriously affect statistics, analysis and decision-making. The classification error is mainly caused by two reasons: The first is due to human factors such as cutting or merging misoperation, and erroneous updates to features that have not changed types, such as large areas of farmland updated to houses and roads. The second is that the image does not support the type, and the classification code is not updated according to the field survey results, and the manual interpretation produces a large area of errors. Figure 8 shows that the problem is that the farmland is wrongly represented as the building. The problem in figure 9 is that the object feature is represented as road, while the field photo is railway. 


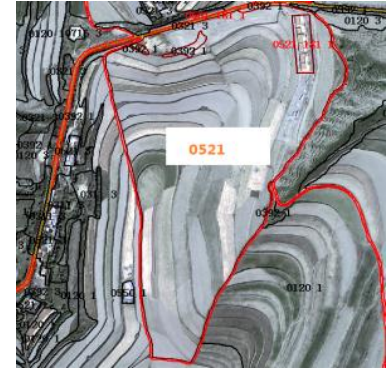

Figure 8. Farmland code error

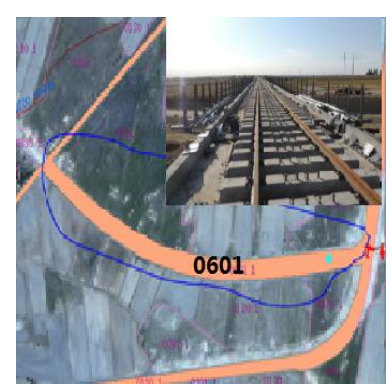

Figure 9. Road code error

\section{QUALITY IMPROVEMENT MEASURESTS}

As the basic data of monitoring, the integrity of image data and thematic data collection and the accuracy of analysis and utilization directly affect the quality of monitoring data.For data production, the main factors affecting its quality include five aspects: personnel, equipment, data, technology and quality control system. The quality improvement of the results of basic geographical condition monitoring should be carried out in these five aspects.

\subsection{Problem-oriented Inspection Method}

The quality control of the data results of geographic national conditions monitoring should adhere to the problem-oriented, shift the inspection focus from the data results to the five aspects of personnel, equipment, data, technology, and quality management system, grasp the key issues, and start with the problems. In the process of inspection, focus on the key links and important factors that seriously affect the quality of the results, including the defects produced in the process where the operator plays a leading role, the reliability of the software used in the automation process, the inadequate data collection and utilization, the deviation of the technical route and method, the poor management and so on. From the practice of monitoring geographical conditions for many years, the most vulnerable link is production. The error-prone problems should be summarized and sorted out, and the quality problems should be analyzed in detail, especially those affecting the application and statistical analysis of the results. According to the result data collection standard, put forward the correct operation method to eliminate the influence of human factors on the data quality problem. In this way, we can accumulate rich operation experience for normal monitoring data production and gradually improve the data quality.

\subsection{Emphasis on Process Quality Control}

On the basis of the traditional quality inspection concept of 'two inspections and one acceptance' of surveying and mapping results, the quality control of geographical national condition monitoring shall be strengthened through the hierarchical quality management from the operation unit to the undertaking unit and then to the national level, move the focus of quality control from results control to process control, strengthen the technical training, supervise and correct technical deviation, timely found that affect the monitoring results of universality, orientation problem, unified technical standards, to ensure data quality conform to the requirements of the technical design. Process quality control is the first and the most important task to ensure the quality management of monitoring results. Process quality control is the most important part of quality management, which is related to the success or failure of quality management. Process quality control will run through the entire production process, including all departments related to production and quality. The effectiveness of the monitoring results can only be guaranteed if the operation department have done a good job in each link of quality training, thematic data, technical design, first-piece result verification, change information collection, process inspection, field investigation, editing and sorting, and final inspection. The accuracy of the data is the lifeline of the monitoring, which is related to the final success or failure of the monitoring of geographical conditions. By carrying out whole-process quality control, the authenticity and reliability of data results can be fundamentally guaranteed, potential risks in the implementation process can be avoided, quality problems can be traceable, the quality of monitoring results can be guaranteed to meet the requirements, and data services can be provided to the country.According to the practice of monitoring the basic geographical conditions for many years, it is the production link that is most likely to cause problems. The error-prone problems should be summarized and sorted out, and the quality problems should be analyzed in detail, especially the problems affecting the application and statistical analysis of the results.

\subsection{Geographical Conditions + Big Data}

With the in-depth development of the investigation and monitoring work in various industries, the scale of various image sources, thematic data and various data products acquired over the years keeps expanding (Liu, J.P., 2019). From the perspective of data volume, growth rate, accuracy and application value, the monitoring data has entered the era of big data (Zhang, J.X., 2016). Quality inspection department should be take advantage of the shared data resources to carry out the quality control method of scientific and technological innovation. Cloud computing is an innovative technology architecture based on the key public information infrastructure of cloud technology, cloud resources, cloud management and cloud services. It provides overall services of information infrastructure by standardizing a large number of service resources such as computing, storage, broadband and software. With the technical support of Internet of things and cloud model, we will explore the integration and sharing of big data of geographical conditions. The use of big data, accurate analysis, to explore the big data environment of fundamental geographical conditions monitoring results test of new ideas. Build a quality control big data support database that can integrate the data from multiple sources. This database is a data pool for users to upload and download, it contains interpretation samples, expert knowledge, and massive change information model extracted by deep learning of big data. Use it to assist quality control, so as to improve quality control efficiency and achieve scientific, impartial, comprehensive and objective.

\section{CONCLUSION}

Fundamental geographic national conditions monitoring is a regular activity, which has provided a large number of reliable data for China's economic and social development. The achievements have been applied in the integration of urban planning, environmental protection and and space use control. The age of big data has arrived, with the integration and unification of natural resources business, various business work will put forward more and higher requirements on geographic information products. It is an important task to analyze the current situation of the quality of the monitoring results and take effective measures to improve the quality. The quality 
improvement method will focus on the development of geographical and national conditions monitoring technology level, diversified results, Internet + big data, etc.

\section{REFERENCES}

Chen, H.Q., He, W.B., Xue, Z.G., 2018: Brief Introduction to the Contents and Methods for Quality Control of Outputs from Geographical Conditions Monitoring. Standardization of Surveying and Mapping 34(3), 22-24.

Li, D.R., Ma, J., Shao, Z.F., 2017: Innovation in the Census and Monitoring of Geographical National Conditions. Journal of Wuhan University 43(1), 2-3.

Liu, J.P., Dong, C., Kang, X.C., Qiu, S.K., Zhao, R., Li, B., Sun, L.J., 2019: National Geographical Conditions Statistical Analysis in the Era of Big Data. Journal of Wuhan University 44(1), 68-69.

Li, Y.L., Guan, L.P., Ding, G.L., 2017: Key Points of Data Updating during National Fundamental Geographic Conditions Monitoring. Standardization of Surveying and Mapping 33(2), 14-15.

Shi, W.Z. et al., 2013: Mdthods and Technologies of National Georaphic State Monitoring. Science Press, 23-32.

Yang, Y., Su, Z.X., Li, D.H., 2019: On the Application of Geographic Conditions Monitoring Results in Natural Resources Ecological Quality Evaluation System. Standardization of Surveying and Mapping 35(4), 16-17.

Zhang, J.X., Gu, H.Y., Lu, X.J., Hou, W., Yu, F., 2016: Research Framework of Geographical Conditions and Big Data. Journal of Remote Sensing 20(5) ,1017-1021. 